\title{
Position control for hybrid infinite-continuous hyper-redundant robot
}

\author{
Cristina Pană ${ }^{1, *}$, Cristian Vladu ${ }^{2}$, Daniela Pătrașcu-Pană ${ }^{1}$, Florina Besnea (Petcu) ${ }^{1}$, Ștefan \\ Cismaru $^{1}$, Andrei Trășculescu ${ }^{1}$, Ionuț Reșceanu ${ }^{1}$, and Nicu Bîzdoacă ${ }^{1}$ \\ ${ }^{1}$ University of Craiova, Faculty of Automation, Computers and Electronics, Mechatronics and \\ Robotics Department, Blvd. Decebal no. 107, Craiova, Romania \\ ${ }^{2}$ University of Craiova, Faculty of Electrical Engineering, Electromechanical, Environmental, and \\ Computer Science Applied in the Electrical Engineering Department, Blvd. Decebal no. 107, Craiova, \\ Romania
}

\begin{abstract}
This paper presents a new conception and analyzes a hyperredundant continuous robot (continuous style manipulator), drive system, and control strategy. The robot includes ten flexible segments and can be extended to several components as needed. The chosen hyper-redundant robot has a continuous infinite hybrid structure (HHRIC), based on hydraulic control with a rheological element. This system combines the advantage of a joint-level drive with a lightweight construction similar to the base-driven robots. It is suitable for tasks such as wiring in hard-toreach areas (caves, subaccounts, steep areas), transportation of fluids or food to areas affected by natural disasters (people buried under ruins), exploration in difficult areas (speleological research). Generally, the control algorithms for hyper-redundant robots are specific to the robots' constructive particularities to which they have applied and the environment in which they operate. Experimental results validate the proposal robot design and control strategies in virtual reality. As a result, it is concluded that hyper-redundant robots and immersive technologies should play an essential role soon in automated and teleoperation applications.
\end{abstract}

\section{Introduction}

\subsection{Continuous hyper-redundant robot}

The kinematic redundancy of hyper-redundant robots is relatively high that can be even infinite. From a constructive point of view, these robots simulate the appearance of tentacles, snakes, or elephant trunks, that have been studied in different ways for the past 25 years. Hyper-redundant manipulators were formerly referred to under multiple names, such as "swan's neck" in [1], "tentacle" in [2], "very high articulated" [3], etc. The name "hyper-

*Correspondingauthor:cristina.pana@edu.ucv.ro 
redundant" was termed by Chirikjian and Burdick [4-7]. Due to their highly articulated nature, can use these robots in applications of:

- Exploration - In unpredictable environments, there are areas of uncertainty where stepping is unsafe or unknown. A hyper redundant device (such as a tentacle, snake) can distribute its entire mass over a wide area of support. Its support between the safety points ensures the continuity of the operation. Such environments comprise planetary and extreme surfaces.

- Inspection- Many inspection techniques in industry and medicine are based on fixedbase mechanisms such as probes, video scopes and endoscopes. These devices are mainly used to inspect cavities that cannot see directly with the naked eye. Such applications include aviation engine maintenance, manufacturing quality control, process monitoring and inspection of chemical utilities and installations.

- Medical - HR-type configurations have also been given attention as potential medical technologies. Minimally invasive surgery reduces or eliminates the opening of large sections of skin or tissue. This thing would have a significant impact in lowering hospitalization time, recovery time and patient suffering and significantly reducing the cost.

- Environments with a high degree of danger - Human activity should be minimal in many areas with high radiation, very high or very low temperatures, toxicity, high pressures or structures with a high degree of fragility (resulting from natural or non-natural disasters). Various wheeled or towed machines have been built for such applications, but there are significant limitations on their ability to cross or manoeuvre in such dangerous terrain. A hyper redundant mechanism may be far more helpful due to the advantages mentioned above. In earthquakes, explosions, etc., the search for possible survivors or the removal of materials is often difficult due to debris and rubble, but an HR system could penetrate such an environment. These are applications that would eliminate the risk of accidents when using heavy construction equipment to dispose of ruins in rugged environments. Other applications would be in mine accidents, tracking inside the cavity, tiny articulated systems could easily penetrate and perform surveillance, ensuring communication with survivors.

- Recognition - Recognition offers some new applications for HR structures. The ability to command tiny eyes or ears provides attractive opportunities for law enforcement agencies. The penetration of dense vegetation by robots, the tentacle or the serpentine can provide information that could not obtain otherwise.

- Guidance, Direction - A special effort in wiring the various structures is made to guide the cables and lines through narrow passages inside some walls and pipes. For short routes, there are many manual tools for wiring these lines, but there are considerable limitations in the situations in which they are used for long ways. These operations involve lying down and working in somewhat awkward positions. Tentacle, horn or snake devices, for example, perform manoeuvres through which they initially pull specific instruments, which are then used to insert the respective cables.

Despite the desirable features, advantages and applications of HR robots, there are still many challenges in manufacturing such robots. To create a truly successful HR robot, all the steps involved in the design need to be evaluated and solved. All this must be thought of as a whole complex.

The implementation details the physical configuration of the HR robot. The format involves selecting the drive system, morphology and mechanical and electronic design, experimental settings and physical modelling.

For each of these stages, the paper further presents an alternative to a new solution proposed and developed by the authors.

At the heart of this framework is a cyclical design process: using the simulation can help the design process. Projects that do not prove to be functional in the simulation stage are not effective in reality either. Similar to the design stage and the choice of drive 
technology may introduce restrictions in the design phase of the robot. Also, simulating the robot's movement using the virtual environment helps establish and obtain good performance in the natural environment. The following paragraph presents some general aspects related to virtual reality and its application in robotics.

\subsection{Virtual Reality}

Although since the 1960s the idea of Virtual Reality (VR) has been around [8], only robotassisted [9-11] and robot-assisted surgery recovery [12-14] have seen widespread use of its usage in robotics. However, there is a strong connection between robotics and Virtual Reality [15]. In Virtual Reality, robots could be employed to offer consumers haptic input to make the experience more engaging $[16,17]$ or to enable the items and the user to interact $[18,19]$. On the other hand, VR provides support on the one hand technology in robotics in the scenario of teleoperation in a virtual environment [20], in the programming of the robot [21], as well as perform research on human-robot contact and collaboration [22-24] and even teach human operators how to cooperate with robots [25].

Modeling and simulation are an essential part of the development of robotic manipulators, in fact, in a variety of other domains. It provides a forum for collaborating with and learning about current and emerging developments in the field. When opposed to a mechanical equivalent, a simulated robot manipulator offers a more cost-effective and scalable approach.

Moreover, VR can enable a large diversity of testing environments, that can be hardly achieved in laboratory conditions. For this reason, the operator can evaluate the robotic structure's behavior much easier, with less effort and at virtually zero cost. Also, this facilitates a lot the training and learning process, ranging from experienced robot operators to university students

\subsection{Structure of the paper}

The topics addressed in this paper are robot dynamics, simulation, and control. The key objective is to monitor the robot's motion using Matlab/Simulink simulation and then by using a virtual robot model.

This following is a description of the paper's structure: The prototype's architecture principles and dynamic model of an infinite-continuous hyper-redundant robot are explained in Section II. Section III introduces the control law and their simulations for a continuous planar hyper-redundant robot. Also, its simulation in a virtual medium is presented. Section IV is dedicated to the conclusions.

\section{Infinite-continuous hyper-redundant robot}

\subsection{Robot system description}

Hyper-redundant robots are developed for operation in areas with limits on mobility and grasping manipulators. They are similar to biological structures like the elephant trunk, snake, octopus tentacle, and others. Constructively, robots have a continuous body, which logically allows for an unlimited number of joints.

Compared to robots operating in open spaces, the location of the body and the effector in the narrow area are often critical for hyper-redundant robots. The load induced by the actuator system (required for each joint) drastically limits their length. 
The robot being analyzed (Fig. 1. a), shown in work [26], solves weight and length problems, maintaining high-performance mobility (at element level).

It is a very long-length, hyper-discrete redundant robot, similar to the snake; its constructive features allow it to address types of movements similar to biological organisms, not so far applicable to hyper-redundant robots (movements of the snake, myriapods, fusiform worms, and others). Such a robot can handle any terrain.

The presented robot consists of identical serial elements (Fig. 1.a). Each component incorporates a four-degree joint consisting of two joint five-degree (Fig. 1.b). The rotational joints are pneumatic cylinders with a controlled rotational movement employing hydraulic rotating cylinders integrated into them. An element of the robot is shown in Fig. 1.b.

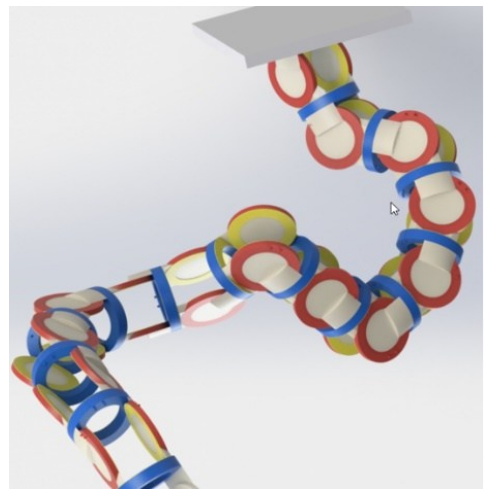

a)

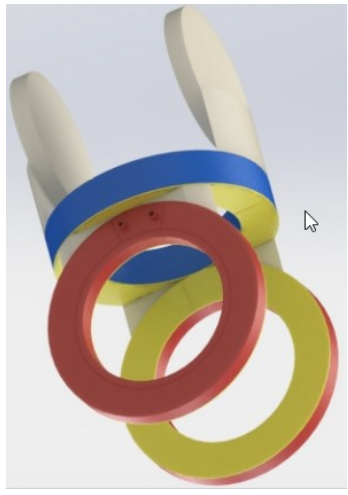

b)

Fig. 1. a) Hybrid Hyper-redundant Robot "Infinitely Continuously" HHRIC - general overview; b) HHRIC - element details

It consists of three pneumatic cylinders of rotation integrated with hydraulic cylinders to control the dynamics of the movement (one cylinder for the longitudinal axis of rotation and two cylinders with unitary movement for the transversal axis of rotation).
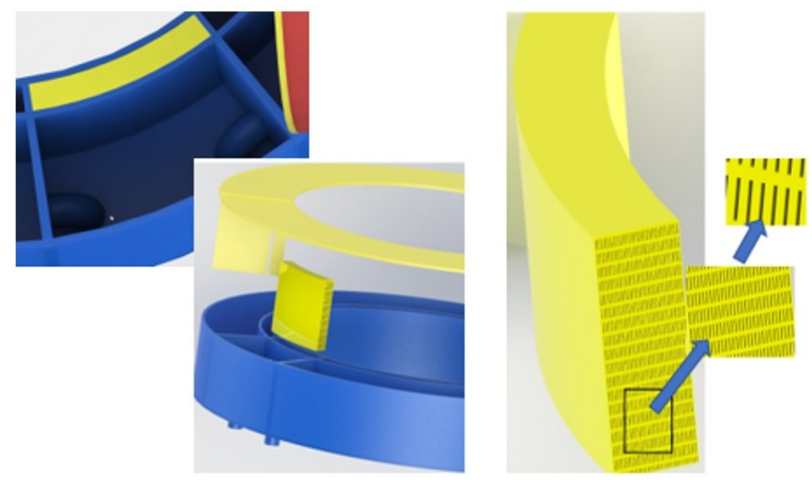

Fig. 2. HHRIC - robot element

By actuating the pneumatic cylinders, the longitudinal or transverse rotation of the element is ensured. The hydraulic motion control cylinders (integrated in the hydraulic cylinders) contains an electrorheological stop valve. By manipulating the electric excitation field's amplitude, the stop valve controls the flow of the fluid, respectively the rotation speed of the hydraulic cylinders. Being unitary with pneumatic cylinders dictates the dynamics of the movement. 
The possible rotation for an element is $300^{\circ}$ longitudinal rotation and $180^{\circ}$ transverse rotation.

For a pressure of 10 bar, a section of 30 elements can be handled by each element (noload).

\subsection{The Lagrange approach for dynamic paradigm of the ideal CHR robot}

The idealized CHR robot will be considered a model as in Fig.3, flexible, in which the internal frictions and damping will be neglected [27]. The effects of the section on the arm dynamics will also be ignored.

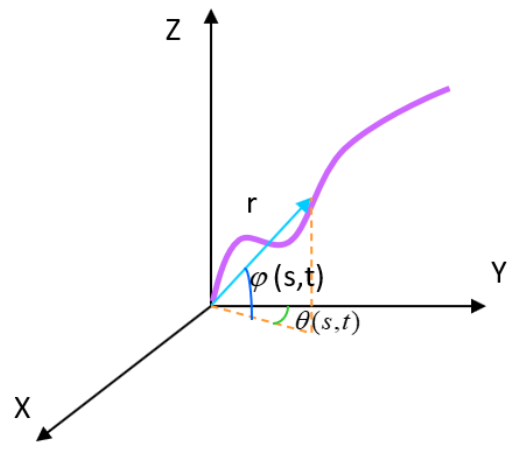

Fig. 3. The ideal model of the CHR robot

The index s is independent and proportional to the length of arc from the curve's origin $\mathrm{C}$, a variable parameter, where

$$
l=l_{0}+\sum_{i=1}^{N} \Delta l_{i}
$$

where $\mathrm{l}_{0}$ denotes the the initial position of the CHR robot's $\mathrm{N}$ components and $\sum_{\mathrm{i}=1}^{\mathrm{N}} \Delta \mathrm{l}_{\mathrm{i}}$ specifies the length of the robot as a control variable. The configuration of the curve $\mathrm{C}$ is based upon two "continuous angles" $\theta(\mathrm{s}), \varphi(\mathrm{s})$ and length variable $\sum_{\mathrm{i}=1}^{\mathrm{N}} \Delta \mathrm{l}_{\mathrm{i}}$.

The robot's orientation, for each point $r(s, t)$, is described through a dextrorotary orthonormal basis vector $\{\mathrm{x}, \mathrm{y}, \mathrm{z}\}$ and its source correspondes to the point $\mathrm{r}(\mathrm{s}, \mathrm{t})$, where $\mathrm{x}$ is tangent to the curve $\mathrm{C}$, while $\mathrm{z}$ is orthogonal to it. The position of a current point $\mathrm{M}$, at moment $t$, at range s originating with the arm, will be described in 3D space by a vector on the associated curve $\mathrm{C}$,

$$
\begin{aligned}
\bar{r} & =\bar{r}(s, t), \quad s \in[0, l] \\
\bar{r}(s, t) & =\left[\begin{array}{lll}
x(s, t) & y(s, t) & z(s, t)
\end{array}\right]
\end{aligned}
$$

where

$$
\begin{gathered}
x(s, t)=\int_{0}^{s} \sin \theta(\sigma, t) \cos \varphi(\sigma, t) d \sigma \\
y(s, t)=\int_{0}^{s} \cos \theta(\sigma, t) \cos \varphi(\sigma, t) d \sigma \\
z(s, t)=\int_{0}^{s} \sin \varphi(\sigma, t) d \sigma, \sigma \in[0, s]
\end{gathered}
$$

The two angular parameters $\theta(\mathrm{s}, \mathrm{t})$ and $\varphi(\mathrm{s}, \mathrm{t})$ establish the size of the arm and ensure the proper positioning of the terminal element: 


$$
\begin{gathered}
x_{L}(t)=x(l, t)=\int_{0}^{l} \sin \theta\left(\sigma^{\prime}, t\right) \cos \varphi\left(\sigma^{\prime}, t\right) d \sigma^{\prime} \\
y_{L}(t)=y(l, t)=\int_{0}^{l} \cos \theta\left(\sigma^{\prime}, t\right) \cos \varphi\left(\sigma^{\prime}, t\right) d \sigma^{\prime} \\
z_{L}(t)=z(l, t)=\int_{0}^{l} \sin \varphi\left(\sigma^{\prime}, t\right) d \sigma^{\prime}
\end{gathered}
$$

The dynamic analysis of the system requires the introduction of the mass element $\mathrm{dm}$ as follows:

$$
d m=\rho d s
$$

where $\rho$ will represent the assumed linear mass density evenly distributed over the length of the CHR robot.

The following notations will also be used:

$$
\begin{gathered}
\varphi=\varphi(s, t), \quad s \in[0, l], t \in\left[0, t_{f}\right] \\
\theta=\theta(s, t), \quad s \in[0, l], t \in\left[0, t_{f}\right] \\
\varphi^{\prime}=\varphi\left(s^{\prime}, t\right), \quad s^{\prime} \in[0, s], t \in\left[0, t_{f}\right] \\
\dot{\varphi}=\frac{\partial \varphi(s, t)}{\partial t}, \quad s \in[0, l], t \in\left[0, t_{f}\right] \\
\dot{\varphi}^{\prime}=\frac{\partial \varphi\left(s^{\prime}, t\right)}{\partial t}, \\
\ddot{\varphi^{\prime}}=\frac{\partial^{2} \varphi\left(s^{\prime}, t\right)}{\partial t^{2}}, \quad s^{\prime} \in[0, s], t \in\left[0, t_{f}\right] \\
F_{\varphi}=F_{\varphi}(s, t), \quad s \in[0, s], t \in\left[0, t_{f}\right], \\
F_{\theta}=F_{\theta}(s, t), \quad s \in[0, l], t \in\left[0, t_{f}\right],
\end{gathered}
$$

where $F_{\varphi}$ and $F_{\theta}$ will represent the distributed forces acting in the planes $\varphi$ and $\theta$, respectively, determine the evolution of the shape of the CHR robot.

According to the position coordinates, the speed at each point, along the arm, will be defined by:

$$
\bar{v}=\left[\begin{array}{lll}
v_{x} & v_{y} & v_{z}
\end{array}\right]
$$

where

$$
\begin{gathered}
v_{x}=\int_{0}^{s}\left(-\dot{\varphi}^{\prime} \sin \varphi^{\prime} \sin \theta^{\prime}+\theta^{\prime} \cos \varphi^{\prime} \cos \theta^{\prime}\right) d \sigma^{\prime} \\
v_{y}=\int_{0}^{s}\left(-\dot{\varphi}^{\prime} \sin \varphi^{\prime} \sin \theta^{\prime}-\theta^{\prime} \cos \varphi^{\prime} \sin \theta^{\prime}\right) d \sigma^{\prime} \\
v_{z}=\int_{0}^{s} \dot{\varphi}^{\prime} \cos \varphi^{\prime} d \sigma^{\prime}
\end{gathered}
$$

Determining the equations of state that govern the motion of a CHR robot is a highly complicated matter. The continuous structure of the model, the existing nonlinearities, the force's and variable's distribution along the arm's length make it difficult to apply conventional methods. In the following, we will obtain the state equations by using 
expanded Lagrange formulas to solve problems involving boundless dimensional systems, that necessitates the determination of the robot's mechanical energies. For a dm element, the kinetic and potential energy will be:

$$
\begin{aligned}
& d E_{\text {cin }}=\frac{1}{2} d m \cdot v^{2} \\
& d E_{\text {pot }}=d m \cdot g \cdot z
\end{aligned}
$$

where

$$
v^{2}=v_{x}^{2}+v_{y}^{2}+v_{z}^{2}
$$

The total energies of the arm will be obtained by integrating the relations (11), (12) with the corresponding substitution of the quantities defined by the expressions (5) and (10):

$$
\begin{gathered}
E_{\text {cin }}=\frac{1}{2} \rho \int_{0}^{l}\left\{\left[\int_{0}^{s}\left(-\dot{\varphi}^{\prime} \sin \varphi^{\prime} \sin \theta^{\prime}+\theta^{\prime} \cos \varphi^{\prime} \cos \theta^{\prime}\right) d \sigma^{\prime}\right]^{2}+\left[\int _ { 0 } ^ { s } \left(-\dot{\varphi}^{\prime} \sin \varphi^{\prime} \sin \theta^{\prime}-\right.\right.\right. \\
\left.\left.\left.\theta^{\prime} \cos \varphi^{\prime} \sin \theta^{\prime}\right) d \sigma^{\prime}\right]^{2}+\left[\int_{0}^{s} \dot{\varphi}^{\prime} \cos \varphi^{\prime} d \sigma^{\prime}\right]^{2}\right\} \\
E_{\text {pot }}=\rho g \int_{0}^{l} \int_{0}^{s} \sin \varphi^{\prime} d \sigma^{\prime} d \sigma
\end{gathered}
$$

To obtain the mathematical model, these expressions will be substituted in Lagrange's equations adopted for infinitely dimensional systems [28, 29]:

$$
\begin{aligned}
& \frac{d}{d t}\left(\frac{\partial E_{\text {cin }}}{\partial \dot{\varphi}}\right)-\left(\frac{\partial E_{\text {cin }}}{\partial \varphi}\right)+\frac{\partial E_{p o t}}{\partial \varphi}=F_{\varphi} \\
& \frac{d}{d t}\left(\frac{\partial E_{c i n}}{\partial \dot{\theta}}\right)-\left(\frac{\partial E_{c i n}}{\partial \theta}\right)+\frac{\partial E_{p o t}}{\partial \theta}=F_{\theta}
\end{aligned}
$$

where $\left(\frac{\partial E_{\operatorname{cin}}}{\partial \dot{\varphi}}\right)$ and $\left(\frac{\partial E_{c i n}}{\partial \dot{\theta}}\right)$ corresponds to the functional partial derivatives (in the Gateaux sense) which are described as the fluctuation of the functional $E_{c i n}$ in respect $\dot{\varphi}$ to the point $s \in[0, l]$. For example, $\left(\frac{\partial E_{c i n}}{\partial \dot{\varphi}}\right)$ has the following form:

$$
\begin{gathered}
\frac{\partial E_{\operatorname{cin}}}{\partial \dot{\varphi}}=\rho\left\{\left[\int_{0}^{s}\left(-\dot{\varphi}^{\prime} \sin \varphi^{\prime} \sin \theta^{\prime}+\theta^{\prime} \cos \varphi^{\prime} \cos \theta^{\prime}\right) d \sigma^{\prime}\right]\left[\int_{0}^{s}\left(-\sin \varphi^{\prime} \sin \theta^{\prime}\right) d \sigma^{\prime}\right]+\right. \\
{\left[\int_{0}^{s}\left(-\dot{\varphi}^{\prime} \sin \varphi^{\prime} \cos \theta^{\prime}-\theta^{\prime} \cos \varphi^{\prime} \sin \theta^{\prime}\right) d \sigma^{\prime}\right]\left[\int_{0}^{s}\left(-\sin \varphi^{\prime} \cos \theta^{\prime}\right) d \sigma^{\prime}\right]+} \\
\left.\left[\int_{0}^{s} \dot{\varphi}^{\prime} \cos \varphi^{\prime} d \sigma^{\prime}\right]\left[\int_{0}^{s}\left(\cos \varphi^{\prime}\right) d \sigma^{\prime}\right]\right\}
\end{gathered}
$$

The other partial derivatives are similarly calculated. Substituting these results in Lagrange's equations, we obtain the final form of the mathematical model:

$$
\begin{gathered}
\rho \int_{0}^{s} \int_{0}^{s}\left\{\ddot{\varphi^{\prime}}\left[\sin \varphi^{\prime} \sin \varphi^{\prime \prime} \cos \left(\varphi^{\prime}-\varphi^{\prime \prime}\right)+\cos \varphi^{\prime} \cos \varphi^{\prime \prime}\right]-\ddot{\theta}^{\prime}\left[\cos \varphi^{\prime} \sin \varphi^{\prime \prime} \sin \left(\theta^{\prime}-\theta^{\prime \prime}\right)\right]+\right. \\
\dot{\varphi}^{\prime 2}\left[\cos \varphi^{\prime} \sin \varphi^{\prime \prime} \cos \left(\theta^{\prime}-\theta^{\prime \prime}\right)-\sin \varphi^{\prime} \cos \varphi^{\prime \prime}\right]+\dot{\theta}^{\prime}\left[\cos \varphi^{\prime} \sin \varphi^{\prime \prime} \cos \left(\theta^{\prime}-\theta^{\prime \prime}\right)\right]+ \\
\left.\dot{\varphi}^{\prime} \dot{\varphi}^{\prime \prime} \sin \left(\varphi^{\prime}-\varphi^{\prime \prime}\right)\right\} d \sigma^{\prime} d \sigma^{\prime \prime}+\rho g \int_{0}^{s} \cos \varphi^{\prime} d \sigma^{\prime}=F_{\varphi} \\
\rho \int_{0}^{s} \int_{0}^{s}\left\{\ddot{\varphi}^{\prime} \sin \varphi^{\prime} \cos \varphi^{\prime \prime} \sin \left(\theta^{\prime}-\theta^{\prime \prime}\right)+\ddot{\theta}^{\prime} \cos \varphi^{\prime} \cos \varphi^{\prime \prime} \cos \left(\theta^{\prime}-\theta^{\prime \prime}\right)-\right. \\
\dot{\varphi}^{\prime 2} \cos \varphi^{\prime} \sin \varphi^{\prime \prime} \sin \left(\theta^{\prime}-\theta^{\prime \prime}\right)+\dot{\theta}^{\prime} \cos \varphi^{\prime} \sin \varphi^{\prime \prime} \sin \left(\theta^{\prime}-\theta^{\prime \prime}\right)+\dot{\varphi}^{\prime} \dot{\theta}^{\prime} \sin \varphi^{\prime} \cos \varphi^{\prime \prime} \cos \left(\theta^{\prime}-\right. \\
\left.\left.\theta^{\prime \prime}\right)\right\} d \sigma^{\prime} d \sigma^{\prime \prime}=F_{\theta}
\end{gathered}
$$

Equations (19) and (20) can be considered equations of an ideal CHR robot's state. We remind that in defining this model, friction and the effects of internal depreciation were neglected. Even with these simplifications, the respective equations represent an integral- 
differential, nonlinear solid model that will raise particular issues in approaching the management problems.

In many applications, both to simplify treatment and address specific cases, the dynamic $3 \mathrm{D}$ spatial model is replaced by a $2 \mathrm{D}$ planar model, such as a robot operating in the OYZ plane. In this case, the primary variable is represented by the angle among the tangent to the inflect and the OY axis,

$$
\varphi=\varphi(s, t)
$$

The dynamic model will be obtained by customizing the model (19) - (21) and will have the form:

$$
\rho \iint\left[\ddot{\varphi}^{\prime} \cos \left(\varphi^{\prime \prime}-\varphi^{\prime}\right)+\dot{\varphi}^{\prime 2} \sin \left(\varphi^{\prime \prime}-\varphi^{\prime}\right)\right] d \sigma^{\prime} d \sigma^{\prime \prime}+\rho g \int_{0}^{s} \cos \varphi^{\prime} d \sigma^{\prime}=F
$$

where $\mathrm{F}$ is the generalized force that determines the change of shape, movement, CHR robot in the OYZ plane of the variable $\varphi$.

\section{Dynamic control for a hyper-redundant continuous robot}

\subsection{Control laws}

The mathematical model of the CHR robot (from the above section) clearly shows the difficulty of establishing an appropriate driving law due to the complexity of the equations that govern a hyper-redundant structure's motion. With nonlinear components challenging to treat, the integrodifferential equations pose particular problems in determining appropriate solutions for the system's management to obtain the required performance.

This section deals with control through ER actuators by using these models' fundamental energy relationships and minimizing the intricacy introduced by the dynamic model's complexity. The energy-based controller [30-32] uses control laws only based on the relationships that define the system's energy. This technique introduces a class of controllers, estabilshing the robot's movement in the desired position with good performance. The procedure is examined for a probing spatial infinite-continuous hyperredundant manipulator, and the regualtory laws are numerically reproduced.

Fig. $4 \mathrm{a}$ and $\mathrm{b}$ present an ideal CHR robot, with a constant distributed moment, with optimal adaptability, and the ability to take on any shape in the operating space.

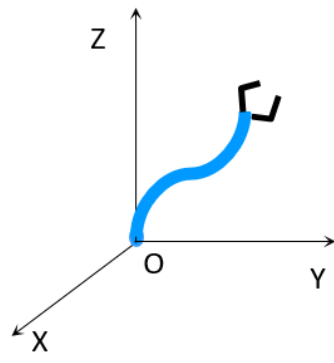

a)

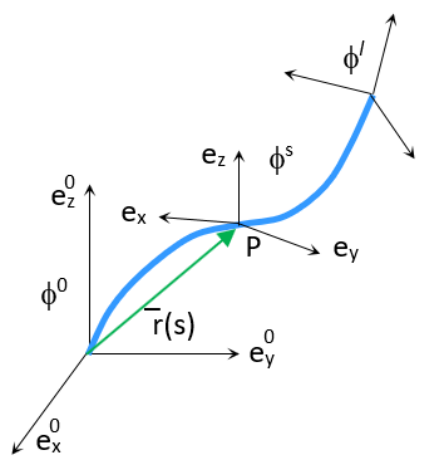

b)

Fig. 4. Infinite-continuous hyper-redundant robot's parameters (ideal model).

Sometimes, conventional approaches are inapplicable in hyper redundant configurations; a huge number of parameters, theoretically unlimited number, and the 
dynamic model's sophistication make it extremely challenging to use traditional algorithms to acquire the control law. The dynamic mathematical problem of a spatial CHR robot have been presented in the above section.

This chapter will expand a dynamic control law focused on the primary energy-work relationship that capable of maintaining the system's closed-loop stability [33, 34]. This approach removes the more complicated issues that derive from the integral derivative model's nonlinearity and offers an easy solution for implementing a suitable controller. A control system is proposed to attain the target posture less using the CHR robot's mathematical model pointed schematically in Fig. 5.

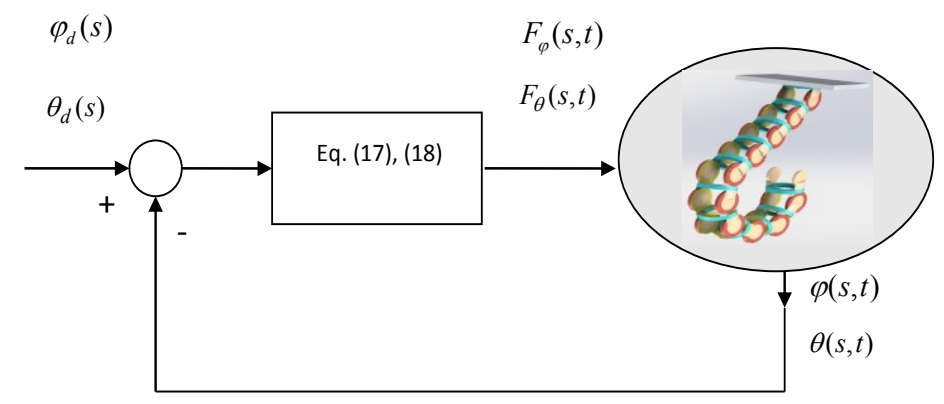

Fig. 5. The general control system of CHR robot.

CHR robot's position control means controlling its movement to achieve the desired position defined by the curve:

$$
C_{d}:\left(\theta_{d}(s), \varphi_{d}(s)\right), \quad s \in[0, l]
$$

The error of the control system will be defined by:

$$
\begin{gathered}
e_{\theta}=\theta(t, s)-\theta_{d}(s) \quad s \in[0, l] \\
e_{\varphi}=\varphi(t, s)-\varphi_{d}(s) \quad s \in[0, l]
\end{gathered}
$$

Next, two control laws will be proposed for the CHR robot operated with electrorheological actuators:

- The first synthesizes the evenly distributed PD leadership, and

- The second synthesizes the evenly distributed PD leadership with spatial weighting

The robot movement's stability in control by each of these laws is demonstrated by the second Lyapunov method in a unique construction.

First, a formal control law is proposed,

$$
\begin{gathered}
F_{\theta}(s, t)=-k_{\theta}^{1}(s) e_{\theta}(s, t)-k_{\theta}^{2}(s) \dot{e}_{\theta}(s, t) \\
F_{\varphi}(s, t)=-k_{\varphi}^{1}(s) e_{\varphi}(s, t)-k_{\varphi}^{2}(s) \dot{e}_{\varphi}(s, t)
\end{gathered}
$$

where $\mathrm{k}_{\theta}^{1}(\mathrm{~s}), \mathrm{k}_{\theta}^{2}(\mathrm{~s}), \mathrm{k}_{\varphi}^{1}(\mathrm{~s}), \mathrm{k}_{\varphi}^{2}(\mathrm{~s})$ are positive coefficients.

In this form, the relations (21) represent a classical PD-type conduction law in which the generated conduction quantity forces are evenly distributed along with the CHR robot.

To appreciate this driving system's quality, we will not resort to the classical solution involving the extremely complicated dynamic model of the robot. Still, we will use the Lyapunov method in a particular construction [35, 36, 29].

The following Lyapunov function will be proposed: 


$$
V(t)=E_{c i n}(t)+E_{\text {pot }}(t)+\frac{1}{2} \int_{0}^{l}\left[k_{\theta}^{1}(s) e_{\theta}^{2}(s, t)+k_{\varphi}^{1}(s) e_{\varphi}^{2}(s, t)\right] d s
$$

$V(t)$ is positively defined because the terms representing kinetic energy and potential energy are positive or zero:

$$
E_{\text {cin }}(t) \geq 0, E_{\text {pot }}(t) \geq 0
$$

Taking into account the energy relations established in the previous paragraph, the derivative of the function (24) will have the form:

$$
\begin{gathered}
\dot{V}(t)= \\
\int_{0}^{l}\left[F_{\theta}(s, t) \dot{e}_{\theta}(s, t)+F_{\varphi}(s, t) \dot{e}_{\varphi}(s, t)+k_{\theta}^{1}(s) e_{\theta}(s, t) \dot{e}_{\theta}(s, t)+\right. \\
\left.k_{\varphi}^{1}(s) e_{\varphi}(s, t) \dot{e}_{\varphi}(s, t)\right] d s
\end{gathered}
$$

Alternatively, replacing the expressions of the control functions, from (23), it results:

$$
\dot{V}(t)=-\int_{0}^{l}\left[k_{\theta}^{1}(s)\left[\dot{e}_{\theta}(s, t)\right]^{2}+k_{\varphi}^{1}(s)\left[\dot{e}_{\varphi}(s, t)\right]^{2}\right] d s \leq 0
$$

which shows the steadiness of the control system and the error's advancement toward zero.

A more efficient control law proposed for situations in which the curve defines the desired position of the movement does not have a "smooth" shape, presenting numerous "corners" and discontinuity elements. Thus, the control law will be in the form of:

$$
\begin{gathered}
F_{\theta}(s, t)=-k_{\theta}^{1}(s) e_{\theta}(s, t)-k_{\theta}^{2}(s) \dot{e}_{\theta}(s, t)-k_{\theta}^{3}(s) f_{\theta}(s, t) \int_{0}^{t} f_{\theta}(s, \tau) \dot{e}_{\theta}(s, \tau) d \tau \\
F_{\varphi}(s, t)=-k_{\varphi}^{1}(s) e_{\varphi}(s, t)-k_{\varphi}^{2}(s) \dot{e}_{\varphi}(s, t)-k_{\varphi}^{3}(s) f_{\varphi}(s, t) \int_{0}^{t} f_{\varphi}(s, \tau) \dot{e}_{\varphi}(s, \tau) d \tau
\end{gathered}
$$

where $\mathrm{k}_{\theta}^{1}(\mathrm{~s}), \mathrm{k}_{\theta}^{2}(\mathrm{~s}), \mathrm{k}_{\theta}^{3}(\mathrm{~s}), \mathrm{k}_{\varphi}^{1}(\mathrm{~s}), \mathrm{k}_{\varphi}^{2}(\mathrm{~s}), \mathrm{k}_{\varphi}^{3}(\mathrm{~s})$ are positive coefficients distributed along with the CHR robot and $\mathrm{f}_{\theta}(\mathrm{s}, \mathrm{t}), \mathrm{f}_{\varphi}(\mathrm{s}, \mathrm{t})$ represent the spatially distributed functions. The third term of the control law introduces a weighting function to facilitate control.

The result was a uniformly distributed PD law with spatial weighting for the hyper redundant system with ER actuation, the stability of which is also demonstrated by the second Lyapunov method.

The following Lyapunov function will be proposed:

$$
\begin{gathered}
V(t)= \\
E_{\text {cin }}(t)+E_{\text {pot }}(t)+ \\
\frac{1}{2} \int_{0}^{l}\left\{k_{\theta}^{1}(s) e_{\theta}^{2}(s, t)+k_{\varphi}^{1}(s) e_{\varphi}^{2}(s, t)+\quad k_{\theta}^{3}(s)\left[\int_{0}^{t} f_{\theta}(s, \tau) \dot{e}_{\theta}(s, \tau) d \tau\right]^{2}+\right. \\
\left.k_{\varphi}^{3}(s)\left[\int_{0}^{t} f_{\varphi}(s, \tau) \dot{e}_{\varphi}(s, \tau) d \tau^{2}\right]\right\} d s
\end{gathered}
$$

$\mathrm{V}(\mathrm{t})$ is positively defined because the terms representing kinetic energy and potential energy are positive or zero:

$$
E_{\text {cin }}(t) \geq 0, E_{\text {pot }}(t) \geq 0
$$

Taking into account the energy relations established in the previous paragraph, the derivative of the function (30) will have the form: 


$$
\begin{gathered}
\dot{V}(t)= \\
\int_{0}^{l}\left[F_{\theta}(s, t) \dot{e}_{\theta}(s, t)+F_{\varphi}(s, t) \dot{e}_{\varphi}(s, t)+k_{\theta}^{1}(s) e_{\theta}(s, t) \dot{e}_{\theta}(s, t)+k_{\varphi}^{1}(s) e_{\varphi}(s, t) \dot{e}_{\varphi}(s, t)+\right. \\
k_{\theta}^{3}(s) \dot{e}_{\theta}(s, t) f_{\theta}(s, t) \int_{0}^{t} f_{\theta}(s, \tau) \dot{e}_{\theta}(s, \tau) d \tau+ \\
\left.k_{\varphi}^{3}(s) \dot{e}_{\varphi}(s, t) f_{\varphi}(s, t) \int_{0}^{t} f_{\varphi}(s, \tau) \dot{e}_{\varphi}(s, \tau) d \tau\right] d s
\end{gathered}
$$

Alternatively, replacing the expressions of the control functions, from (24), it results:

$$
\dot{V}(t)=-\int_{0}^{l}\left[k_{\theta}^{1}(s)\left[\dot{e}_{\theta}(s, t)\right]^{2}+k_{\varphi}^{1}(s)\left[\dot{e}_{\varphi}(s, t)\right]^{2}\right] d s \leq 0
$$

which shows the steadiness of the control system and the error's advancement toward zero.

So we have the following:

- Uniformly distributed PD control: The CHR robot's closed-loop system is reliable if the control law is considered by:

$$
\begin{aligned}
& F_{\theta}(s, t)=-k_{\theta}^{1}(s) e_{\theta}(s, t)-k_{\theta}^{2}(s) \dot{e}_{\theta}(s, t) \\
& F_{\varphi}(s, t)=-k_{\varphi}^{1}(s) e_{\varphi}(s, t)-k_{\varphi}^{2}(s) \dot{e}_{\varphi}(s, t)
\end{aligned}
$$

where $k_{\theta}^{1}(s), k_{\theta}^{2}(s), k_{\varphi}^{1}(s), k_{\varphi}^{2}(s)$ are positive coefficients.

- Spatially distributed control: The CHR robot's closed-loop system is reliable if the control law is considered by:

$$
\begin{aligned}
& F_{\theta}(s, t)=-k_{\theta}^{1}(s) e_{\theta}(s, t)-k_{\theta}^{2}(s) \dot{e}_{\theta}(s, t) \\
- & k_{\theta}^{3}(s) f_{\theta}(s, t) \int_{0}^{t} f_{\theta}(s, \tau) \dot{e}_{\theta}(s, \tau) d \tau F_{\varphi}(s, t) \\
= & -k_{\varphi}^{1}(s) e_{\varphi}(s, t)-k_{\varphi}^{2}(s) \dot{e}_{\varphi}(s, t) \\
- & k_{\varphi}^{3}(s) f_{\varphi}(s, t) \int_{0}^{t} f_{\varphi}(s, \tau) \dot{e}_{\varphi}(s, \tau) d \tau
\end{aligned}
$$

where $\mathrm{k}_{\theta}^{1}(\mathrm{~s}), \mathrm{k}_{\theta}^{2}(\mathrm{~s}), \mathrm{k}_{\theta}^{3}(\mathrm{~s}), \mathrm{k}_{\varphi}^{1}(\mathrm{~s}), \mathrm{k}_{\varphi}^{2}(\mathrm{~s}), \mathrm{k}_{\varphi}^{3}(\mathrm{~s})$ are positive factors distributed in conjunction with the CHR robot and $\mathrm{f}_{\theta}(\mathrm{s}, \mathrm{t}), \mathrm{f}_{\varphi}(\mathrm{s}, \mathrm{t})$ represent the spatially distributed functions.

Since the stability demonstration is not contingent upon the system dynamics, the issues related with model-based controllers [37, 38, 33] are prevented. The control laws (23) and (28) are autonomous of the system indexes, implying that they are stable and robust to the uncertainties associated with these parameters.

The requirement to determine a good sizing of the system increase complication in choosing control parameters $\mathrm{k}_{\theta}^{1}(\mathrm{~s}), \mathrm{k}_{\theta}^{2}(\mathrm{~s}), \mathrm{k}_{\varphi}^{1}(\mathrm{~s}), \mathrm{k}_{\varphi}^{2}(\mathrm{~s})$. Of course, the stability of the closed-loop system demands just that $\mathrm{k}_{\theta}^{1}(\mathrm{~s})>0, \mathrm{k}_{\theta}^{2}(\mathrm{~s})>0, \mathrm{k}_{\varphi}^{1}(\mathrm{~s})>0, \mathrm{k}_{\varphi}^{2}(\mathrm{~s})>0$, but in experiments or simulations, achieving adequate performance requires a calculation procedure. Indeed, this selection of parameters varies according to the sophistication of the dynamic model of the function and the estimation of the control coefficients; the easiest and fastest solution was to use heuristic methods.

An approximation method is proposed for the evaluation of the control parameters in the context of some simplifying hypotheses in which it is assumed that: 
- Hypothesis 1: The movement of the CHR robot corresponds to "small" displacements, which check the condition $[35,36]$ :

$$
\left|\varphi\left(s^{\prime}, t\right)-\varphi\left(s^{\prime \prime}, t\right)\right|<\delta, s^{\prime}, s^{\prime \prime} \in[0, s], t \in\left[0, t_{f}\right]
$$

- Hypothesis 2: Motion control is a sequential control; the CHR robot elements reach the desired position systematically; the first component is positioned in the preferred standing, followed by the second and so forth.

Under the conditions Hypothesis 1, Hypothesis 2, the control system (28), can be estimated in the error space through the equality:

$$
\ddot{e}_{i}+\frac{k_{i}^{2}}{\rho \Delta^{2}} \dot{e}_{i}+\frac{k_{i}^{1}}{\rho \Delta^{2}} e_{i}+\frac{g}{\Delta} h_{i} e_{i}=0, \quad i=\overline{1, N},
$$

where $h_{i}=h_{i}\left(\varphi_{d_{i}}\right)$ denotes the nonlinear term defined in the error space by the gravitational variable, while $\mathrm{k}_{\mathrm{i}}^{1}, \mathrm{k}_{\mathrm{i}}^{2}$ correlate to or in the plane $\varphi$ or accordingly the plane $\theta$.

Equation (34) can be interpreted in conventional terminology of the damping rate $\xi_{\mathrm{i}}$ and the natural frequency $\omega_{n_{i}}$,

$$
\ddot{e}_{i}+2 \xi_{i} \omega_{n_{i}} \dot{e}_{i}+\omega_{n_{i}}^{2} e_{i}+h_{i}^{*} e_{i}=0
$$

where

$$
\begin{aligned}
& \omega_{n_{i}}=\left(\frac{k_{i}^{1}}{\rho \Delta^{2}}\right)^{\frac{1}{2}} \\
& \xi_{i}=\frac{k_{i}^{2}}{2\left(\rho \Delta^{2} k_{i}^{1}\right)^{\frac{1}{2}}} \\
& h_{i}^{*}=\frac{g}{\Delta} h_{i}
\end{aligned}
$$

The use of a sliding control system is suggested for this model in which the direction is compelled to follow the switching axis, which is located immediately at the origin, in the classical terms of the damping rate $\xi_{\mathrm{i}}$, according to Fig. 6.

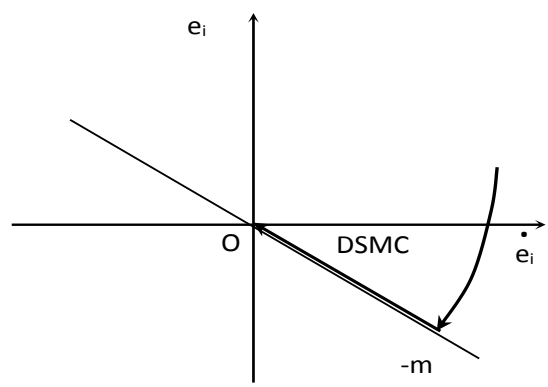

Fig. 6. Direct Sliding Mode Control (DSMC).

This precise control is called DSMC and was introduced in [39] for linear systems. The DSMC control may be acquired in the classic terms of the amortization rate $\xi_{\mathrm{i}}$ that verifies the conditions:

$$
\begin{gathered}
\xi_{i}^{2}>1+\frac{h_{i}^{*}}{\omega_{n_{i}}{ }^{2}} \\
h_{i}^{*}>-\omega_{n_{i}}{ }^{2}
\end{gathered}
$$


The increase of $\xi_{\mathrm{i}}$ determines an over-damped movement, a beneficial phenomenon because this control guarantees the system's overall sturdiness.

- The option of this index is centered on an approximation system, establishing the basic range of control coefficients.

- Functions $f_{\theta}(s, t), f_{\varphi}(s, t)$ can be entered to achieve better performance when the desired trajectory has a shape that is not "smooth", with corners and shapes "sharp". For example, $f_{\theta}, f_{\varphi}$ there may be relationships of the distance between the real standing and the endpoints of the requested trajectory.

\subsection{Simulation}

For a higher comprehension of the control, the CHR planar robot is analyzed in the OZY plan. We use the dynamic model (20) and the control law (23). Spatial discretization is introduced $\mathrm{s}=\mathrm{i} \Delta, \mathrm{i}=\overline{1,10}$.

Using Hypothesis 1, the two relationships can be rewritten in the error space, as follows:

$$
\rho \Delta^{2} \sum_{i=1}^{10} \ddot{e}_{i}+k_{\varphi_{i}}^{2} \dot{e}_{i}+k_{\varphi_{i}}^{1} e_{i}+\rho g \sum_{i=1}^{10} h_{i}\left(\varphi_{d}\right) e_{i}=0
$$

where:

$$
\mathrm{e}_{\mathrm{i}}(\mathrm{t})=\mathrm{e}(\mathrm{i} \Delta, \mathrm{t})=\varphi(\mathrm{i} \Delta, \mathrm{t})-\varphi_{\mathrm{d}}(\mathrm{i} \Delta), \mathrm{h}_{\mathrm{i}}\left(\varphi_{\mathrm{d}}\right)=\left(\frac{\partial \mathrm{H}}{\partial \varphi}\right)_{\varphi=\varphi_{\mathrm{d}}, \mathrm{s}=\mathrm{i} \Delta}, \mathrm{H}(\varphi)=\cos \varphi
$$

A sequential spatial control strategy (Hypothesis A2) causes the global movement to decompose. For the initial element, $1=1$,

$$
\rho \Delta^{2} \ddot{e}_{1}+k_{\varphi_{1}}^{2} \dot{e}_{1}+k_{\varphi_{1}}^{1} e_{1}+\rho g h_{1} e_{1}=0
$$

The control law determines:

$$
\lim _{t \rightarrow \infty} e_{1}=0
$$

This process is repeated for every element, $\mathrm{l}=\overline{2,10}$.

The DSMC control (Figure 4) compel the conditions [15, 24]:

$$
\frac{\dot{e}_{i}}{e_{i}}=-l
$$

On the changing line (44), the criterion for convergence of the movement to zero can be easily determined as follows:

$$
\begin{gathered}
\xi_{i}^{2}>1-\frac{g \sin \varphi_{d_{i}}}{\Delta \omega_{n_{i}}{ }^{2}} \\
\frac{g}{\Delta} \sin \varphi_{d_{i}}<\omega_{n_{i}}{ }^{2}
\end{gathered}
$$

For the simulation test (Fig.7, 8), the initial position of the CHR robot is chosen as the vertical line (OY axis),

case 1: the curve defines the desired position of the movement as a "smooth" shape, which can represent the movement of the robot in a grasping application 


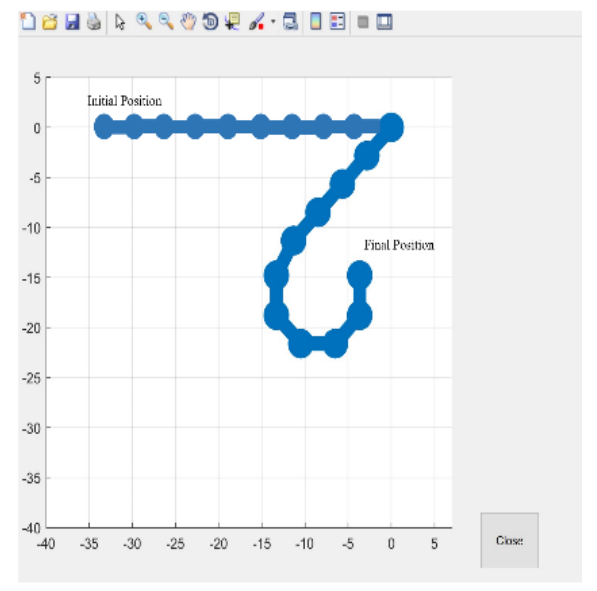

a)

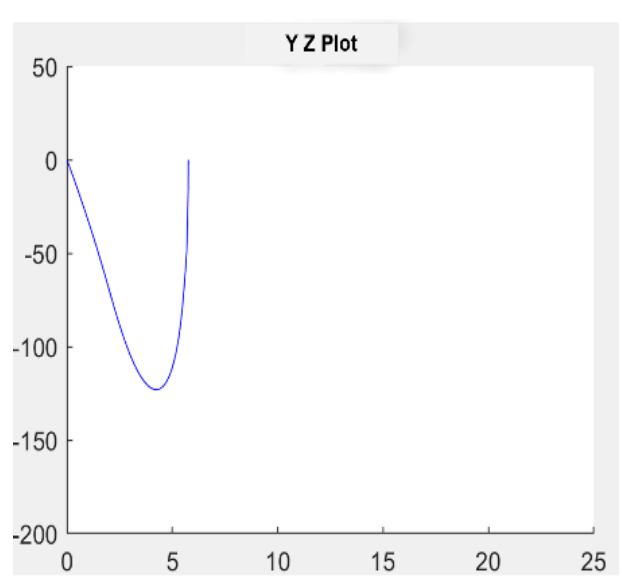

b)

Fig. 7. Simulation results: a) The evolution of the robot CHR to the requested place with the highlighting of both the initial and the final position, b) phase portrait of the error.

case 2: the curve defines the desired position of the movement, presenting numerous "corners" and discontinuity elements (same initial position of the CHR robot), which can represent the movement of the robot in a manipulative application in a narrow and uneven operating environment

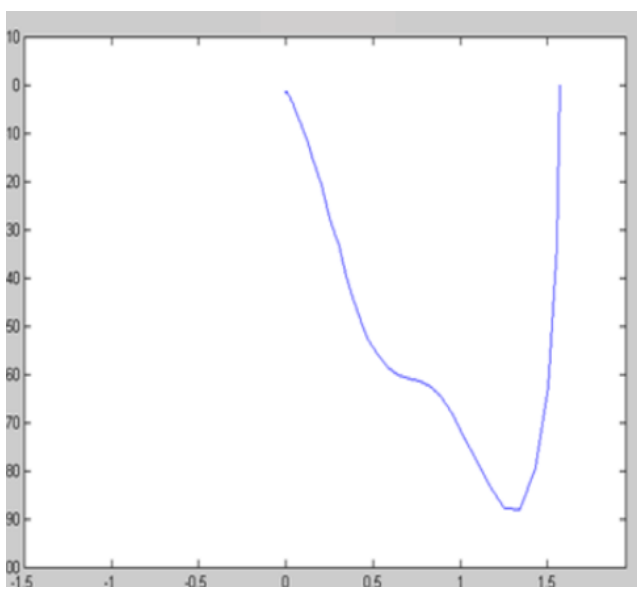

a)

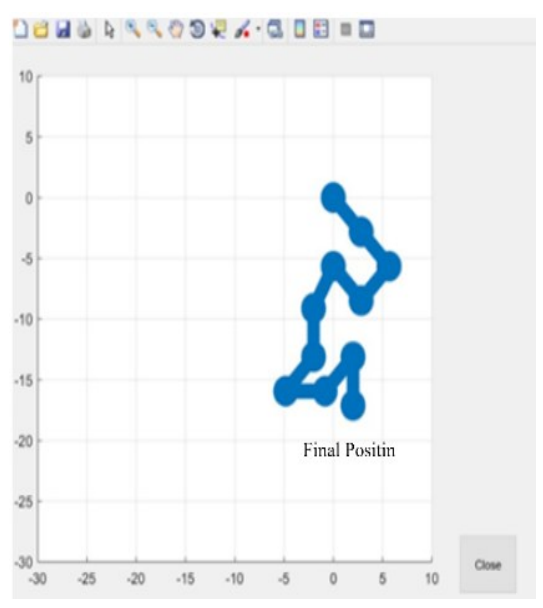

b)

Fig. 8. Simulation results: a) The evolution of the robot CHR to the desired position with the highlight of the final standing, b) phase portrait of the error.

\subsection{Implementation in the virtual environment}

Since developing and testing software for a real robot is expensive and time-consuming, modeling is an essential aspect of the robotic application growth. Reinforcing the application in a prototype before applying it to the robot will reduce the iteration period by identifying possible problems early. Simulating also allows for studying specific cases or situations that would be too risky to examine in a real environment. A full 3D model of the Infinite-CHR to be managed with actual specifics has been constructed and conveniently 
brought into the Unity3D project scene as a.fbx extension or as a Prefab. This assignment was completed with the external CAD and architecture software Solidworks (Fig.9).

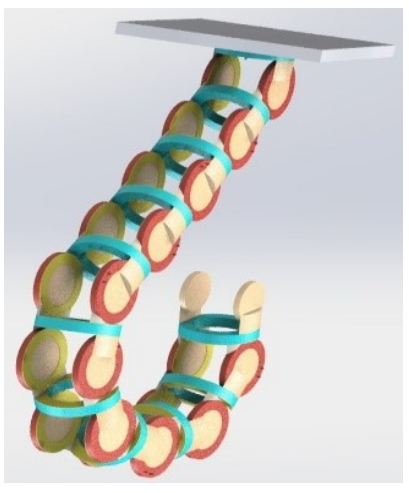

a)

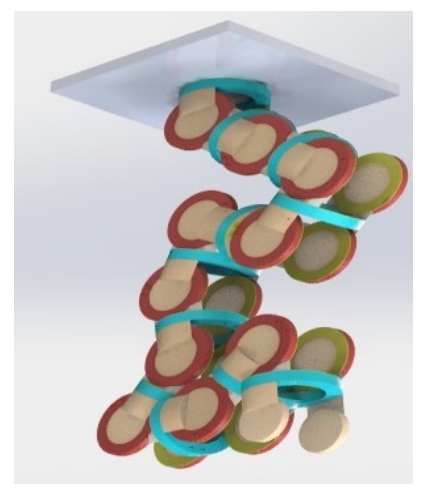

b)

Fig. 9. The Infinite-CHR robot modeled in Solidworks..

The import is validated by visually inspecting the model in the VR environment for any displacements. Besides that, the Unity 3D engine validates the complete migration of mesh details, representing a set of vertices that build the form of the model. No data must be lost during the transition from CAD software to the VR engine. The final goal for integrating such a model in a virtual environment is to provide a future possibility to easily access such a CHR robot to develop practical applications without the need to own one.
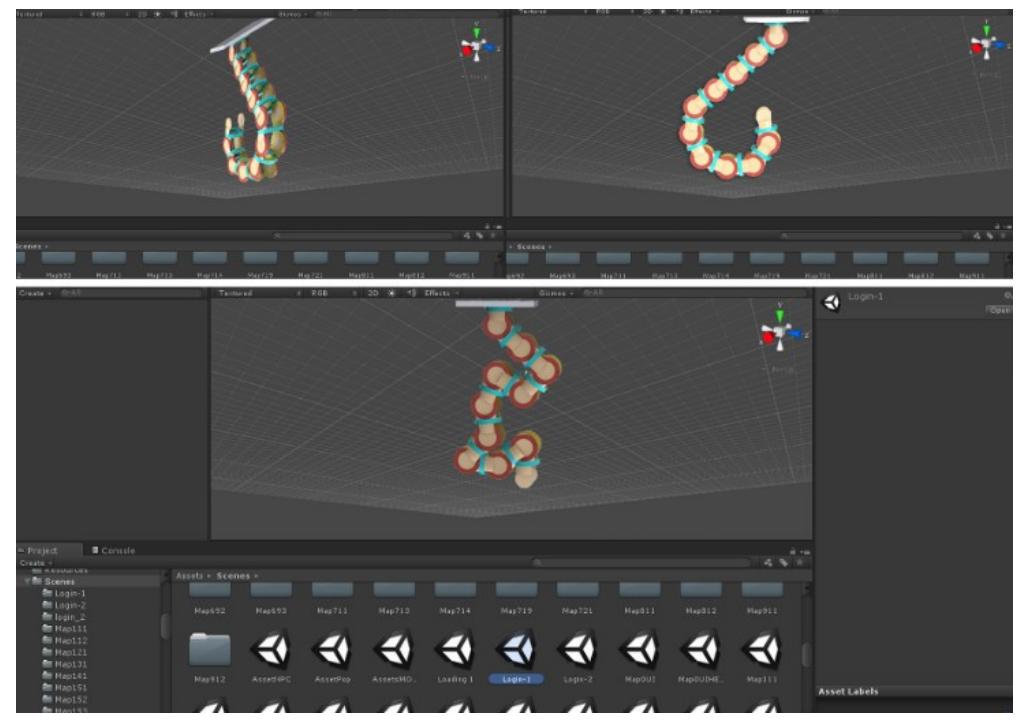

Fig. 10. Unity imported model of the Infinite-CHR robot.

Simulating the CHR robot using the Unity $3 \mathrm{D}$ engine is favorable since Physics can be implemented and simulated. This means that the environment in which the robot is simulated can reach quite realistic parameters. For example, in the scenario the operator can vary the robot's mass, friction, acceleration and other such parameters that can gratly influence the dynamics of the robot. Also, by varying these parameters, the aging of the robot can be simulated, and also what happens if one of the robot's joints fails or is out of its typical parameters. 


\section{Conclusions}

The new approach to continuous robot control introduced in this work is motivated by reducing computational complexity. The main innovation is both the robot prototype presented and a general control strategy based on energies. This control strategy is then implemented on a virtual robot. Thus, the virtual robot is selected as subject to directly map his degrees of freedom to those of the hyper-redundant continuous robot for which control is desired.

Acknowledgments: This work was supported by the grant POCU/380/6/13/123990, „Entrepreneurial University - system of higher education and training for the Romanian labor market by awarding scholarships for Ph.D. students and postdoctoral researchers and the implementation of programs innovative entrepreneurial training", co-financed by the European Social Fund within the Sectorial Operational Program Human Capital 2014 - 2020 and by the grant of the Romanian Ministry of Education and Research, CCCDI - UEFISCDI, project number PN-III-P2-2.1-PED-20190937, within PNCDI III.

\section{References}

1. V.V. Anderson, R.C. Horn, ASME Trans. 67-DE-57, 1-12, (1967)

2. G.S. Chirikjian, Theory and Applications of Hyper-Redundant Robotic Mechanisms, (Ph.D Dissertation, Department of Engineering and Applied Science, California Institute of Technology, 1992).

3. G.S. Chirikjian, J.W. Burdick, IEEE Int. Conf. Robot. Autom. (1990)

4. J.W. Burdick, G.S. Chirikjian, IEEE T. Robotic. Autom. 11, 6, 794-805, (1995)

5. G. S. Chirikjian, J. W. Burdick, P. IEEE Int. Conf. Robot. Autom, 625-631, (1990)

6. G. S. Chirikjian, J. W. Burdick, P. IEEE Int. Conf. Robot. and Autom., 415-420, (1992)

7. G. S. Chirikjian, P. IEEE Int. Conf. Robot. Autom., 107-112, (1993)

8. T. Mazuryk, M. Gervautz, Appl. Tech. Future, (1996)

9. J.D. Bric, D.C. Lumbard, M.J. Frelich, J.C. Gould,. Surg. Endosc, 30, 2169-2178 (2016).

10. A. Moglia, V. Ferrari, L. Morelli, M. Ferrari, F. Mosca, A. Cuschieri, Eur. Urol. 69, 1065-1080, (2016)

11. O.A. Van der Meijden, M.P. Schijven, Surg. Endosc., 23, 1180-1190, (2009)

12. S.V. Adamovich, G.G. Fluet, E. Tunik, A.S. Merians, Neuro Rehabil. 25, 29-44, (2009)

13. K. Baur, A. Schättin, E.D. de Bruin, R. Riener, J.E. Duarte, P. Wolf, J. Neuroeng. Rehabil. 15, $107(2018)$

14. M.C. Howard, Comput. Hum. Behav. 70, 317-327, (2017)

15. G.C. Burdea, IEEE Trans. Robot. Autom. 15, 400-410, (1999)

16. M. Al-Sada, K. Jiang, S. Ranade, M. Kalkattawi, T. Nakajima, Virtual Real. 24, 191-209, (2020)

17. E. Vonach, C. Gatterer, H. Kaufmann, P. IEEE Virt. Real., 74-83, (2017)

18. Y. Zhao, L.H. Kim, Y. Wang, M. Le Goc, S. Follmer, P. Interactive Surfaces Spaces, 82-91, (2017)

19. R. Suzuki, H. Hedayati, C. Zheng, J.L. Bohn, D. Szafir, E.Y.L. Do, M.D. Gross, D. Leithinger, Conf. Human Factors in Computing Systems, 1-11, (2020)

20. M. Elobaid, Y. Hu, G. Romualdi, S. Dafarra, J. Babic, D. Pucci, Telexistence and Teleoperation for WalkingHumanoid Robots. In Intelligent Systems and Applications; (Springer International Publishing: Cham, Switzerland, 1106-1121, 2020)

21. G. Bolano, A. Roennau, R. Dillmann, A. Groz, P. In.Conf. U.R.625-630, (2020)

22. O. Liu, D. Rakita, , B. Mutlu, M. Gleicher, P. IEEE Int. S. RO-MAN. 751-757, (2017)

23. V. Villani, B. Capelli, L. Sabattini, P. IEEE Int. S. RO-MAN., 422-427, (2018)

24. L. Wijnen, S. Lemaignan, P. Bremner, P. ACM/IEEE Int. Conf. RO-MAN 514-516, (2020)

25. E. Matsas, G.C. Vosniakos, Int. J. Interact. Des. Manuf. 11, 139-153, (2017)

26. I. Vladu, D. Cojocaru, F. Manta, I. Reşceanu, Int. Conf. Sys. Theory Control Computing (2019)

27. M. Ivănescu, Dynamic Control for a tentacle manipulator, Robotics and Factories of the future, (Springer-Verlag, 317-329, 1985) 
28. I. Gravagne, I.D. Walker, IEEE Int. EEE Int. Conf. Robot. Autom, $2544-2550$, (2000)

29. P. K. C. Wang, Control of distributed parameter systems, in Advance in Control Systems, (Academic Press, 1965)

30. K. B. Shigoma, Int. J. Robo. Research 15, 3, 230 - 266, (1996)

31. G. S. Chirikjian, P. Int. Conf. Intelligent Robo. Systems, 1059 - 1066, (1993)

32. G.S. Chirikjian, Adv. Robo. 9, 3217-243, (1995)

33. S. S.Ge, T. H Lee, G. Zhu, Mechatronics 6, 7, 779 - 798, (1996)

34. Z. P. Wang, S.S. Ge, T.H. Lee, Asian Conf. on Robo. and its Appl., 268 - 273, (2001)

35. N. G. Bîzdoacă, D. Pană, C. Pană, M. Niculescu, Int. Conf Computer Meth. Mechanicsc, (2003)

36. M. Ivănescu, P. IEEE Int. Conf. Robo. Autom., 1531 - 1538, (2002)

37. M. Ivănescu, I. Badea, Dynamic Control for a tentacle manipulator, P. Int. Conf. Robo. Factories Future, 315 327, (1984)

38. M. Ivănescu, V. Stoian, IEEE Int. Conf. Robo. Autom., 3155-3160, (1995)

39. E. Mishkin, Jr L. Braun, Adaptive Control Systems (New York, McGraw Hill, 1961) 